\title{
Electrical Characterization And Study of Current Drift Phenomena And Hysteresis Mechanism In Junctionless Ion-Sensitive Field-Effect Transistor.
}

Jaydeep Singh Parmar ( 2016rec9510@mnit.ac.in )

Malaviya National Institute of Technology https:// orcid.org/0000-0002-0424-8703

Nawaz Shafi

MNIT Jaipur: Malaviya National Institute of Technology

Chitrakant Sahu

MNIT Jaipur: Malaviya National Institute of Technology

\section{Research Article}

Keywords: Drift, Hysteresis, ISFET, Junctionless

Posted Date: June 29th, 2021

DOI: https://doi.org/10.21203/rs.3.rs-618078/v1

License: (c) (i) This work is licensed under a Creative Commons Attribution 4.0 International License.

Read Full License 


\title{
Electrical Characterization and Study of Current Drift phenomena and Hysteresis Mechanism in Junctionless Ion-Sensitive Field-Effect Transistor
}

\author{
Jaydeep Singh Parmar ${ }^{\mathrm{a}}$, Nawaz Shafi, ${ }^{\mathrm{a}, 1}$, Chitrakant Sahu ${ }^{\mathrm{a}, 1,}$ \\ ${ }^{a}$ Dept. of Elec. $\mathcal{E}$ Commn., Malaviya National Institue of Technology, Jaipur
}

\begin{abstract}
A comprehensive study of the drain current drift mechanism and hysteresis phenomena in fabricated p-channel junctionless ion-sensitive field-effect transistor (JL-ISFET) has been investigated for the first time. The current drift measurements have been performed through transient analysis of drain current, under different $\mathrm{pH}$ and liquid-gate bias $\left(V_{l g}\right)$. Further, time-dependent gate-capacitance $\left(C_{G}\right)$ has also been analyzed to see the effect of hydroxyl ions $\left(\mathrm{OH}^{-}\right)$in the sensing film $\left(\mathrm{Al}_{2} \mathrm{O}_{3}\right)$. The hysteresis has also been investigated for different $\mathrm{pH}$ loop $(7 \rightarrow 3 \rightarrow 7 \rightarrow 11 \rightarrow 7$ and $7 \rightarrow 11 \rightarrow 7 \rightarrow 3 \rightarrow 7)$ and times (960s, 1500s, and 1920s). It has been observed that the drift of JL-ISFET occurs because of chemical modification of the sensing film, due to $\mathrm{OH}^{-}$. The proposed device exhibits threshold voltage sensitivity of $58.2 \mathrm{mV} / \mathrm{pH}$ that is near to the Nernstian limit. Further, the hysteresis width and maximum drain current drift are measured as $\sim 1.3 \mathrm{mV}$ and $2.4 \mu \mathrm{A}(\sim 75 \%)$, respectively.
\end{abstract}

Keywords: Drift, Hysteresis, ISFET, Junctionless.

\section{Introduction}

Continuous growth in nano-fabrication technology results in extensive research on biosensors, capable of detecting tiny biological species such as protein, virus, DNA, enzyme, and antibodies $[1,2,3,4,5]$. In recent studies, $\mathrm{pH}$-sensors have been used in different fields, such as environmental condition monitoring, chemical and biological process monitoring, and food manufacturing and processing [6]. Particularly, field-effect transistor (FETs)-based ion-sensitive field-effect transistor (ISFET) have gained interest as a promising candidate to be used as a point-of-care device for sensing applications due to their advantages, including existing CMOS process compatibility, miniaturized sensor size, low power consumption, portability, real-time and label-free detection $[7,8,9,10]$. Despite their advantages, the

Email addresses: 2016rec9510@mnit.ac.in (Jaydeep Singh Parmar), chitrakant.ece@mnit.ac.in (Chitrakant Sahu) 
ISFETs conventionally need ultra-shallow differentially doped junctions between sourcechannel -drain regions, which is very difficult and expensive at smaller nodes.

A non-classical junctionless field-effect transistor (JL-FET) approach have been introduced to overcome the aforementioned issues in conventional ISFETs [11, 12]. JL-FETs have a uniformly doped channel, and their fabrication is easy as compared to conventional MOSFETs, $[13,14]$. Further, Junctionless transistors are governed through bulk conduction instead of surface conduction [15]. In our previous work, we demonstrated the effect of channel width on threshold voltage and drain current sensitivity of p-channel junctionless ISFET (JL-ISFET), with threshold voltage sensitivity near to theoretical Nernstian limit $[16]$.

Despite the better sensitivity of the p-channel JL-ISFET, the accuracy of the measured results in a biosensor, gets affected by the hysteresis and current drift. In this regard, the drain current drift and hysteresis of p-channel JL-ISFET have been investigated for the first time in this paper. The origin of the current drift mechanism has been investigated by measuring drain current $\left(I_{d}\right)$ and gate capacitance $\left(C_{G}\right)$ as a function of time for different $\mathrm{pH}(5,7,9)$ and liquid - gate bias $\left(V_{l g}=-1 \mathrm{~V},-2 \mathrm{~V},-3 \mathrm{~V}\right)$. Further, hysteresis in p-channel JL-ISFET has also been investigated for different loop path $(7 \rightarrow 3 \rightarrow 7 \rightarrow 11 \rightarrow 7$ and $7 \rightarrow$ $11 \rightarrow 7 \rightarrow 3 \rightarrow 7$ ) and loop times (960s, 1500s, and 1920s), to study the impact of hydroxyl ions $\left(\mathrm{OH}^{-}\right)$on the sensing film. Finally, a comparison of the hysteresis width has also been performed for devices with different sensing films. Section II presents the fabrication method and measurement setup of the p-channel JL-ISFET. Section III presents the electrical characterization, drift, and hysteresis analysis of p-channel JL-ISFET in subsections A, B, and $\mathrm{C}$, respectively.

\section{Device Fabrication}

The physical dimensions of the fabricated p-channel JL-ISFET for different layers deposited can be seen in Table 1 .

Table 1: Physical Dimensions of the p-channel JL-ISFET for different layers with their roles in pH sensor investigated .

\begin{tabular}{lll}
\hline Parameter (Role) & Thickness & Dimensions $(\mathbf{L} \times \mathbf{W})$ \\
\hline \hline $\mathrm{Al}_{2} \mathrm{O}_{3}$ (Sensing membrane) & $5 \mathrm{~nm}$ & $30 \times 15 \mu \mathrm{m}$ \\
\hline PolySilicon (device layer) & $50 \mathrm{~nm}$ & $50 \times 15 \mu \mathrm{m}$ \\
\hline $\mathrm{Cr} / \mathrm{Au}$ (Contacts ) & $150 \mathrm{~nm}$ & $100 \mu \mathrm{m} \times 100 \mu \mathrm{m}$ \\
\hline $\mathrm{SiO}_{2}$ (Passivation layer) & $100 \mathrm{~nm}$ & $40 \mu \mathrm{m} \times 40 \mu \mathrm{m}$ \\
\hline $\mathrm{Cr} / \mathrm{Au}$ (Back- gate contact) & $150 \mathrm{~nm}$ & Full wafer $($ Back side) \\
\hline
\end{tabular}

\subsection{Fabrication}

Fabrication process steps have been discussed in detail in [16]. A batch of boron-doped wafer was oxidized for 1 hour, at $1000{ }^{\circ} \mathrm{C}$ in the furnace to grow $50 \mathrm{~nm} \mathrm{SiO}$. Further, 


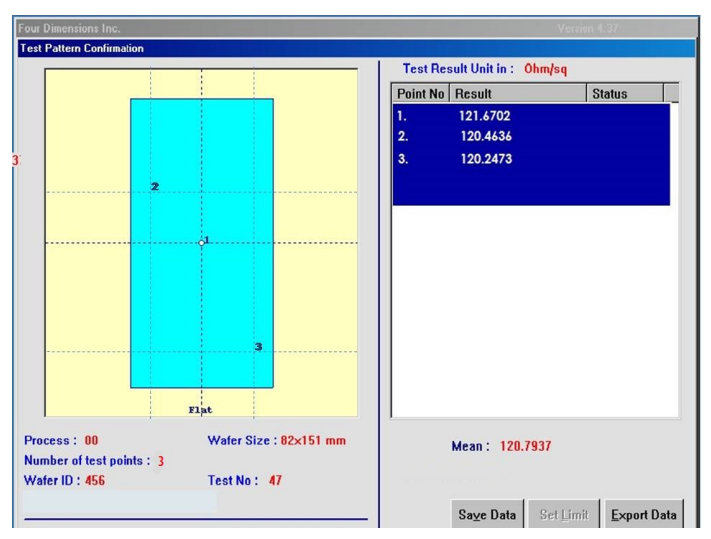

(a)

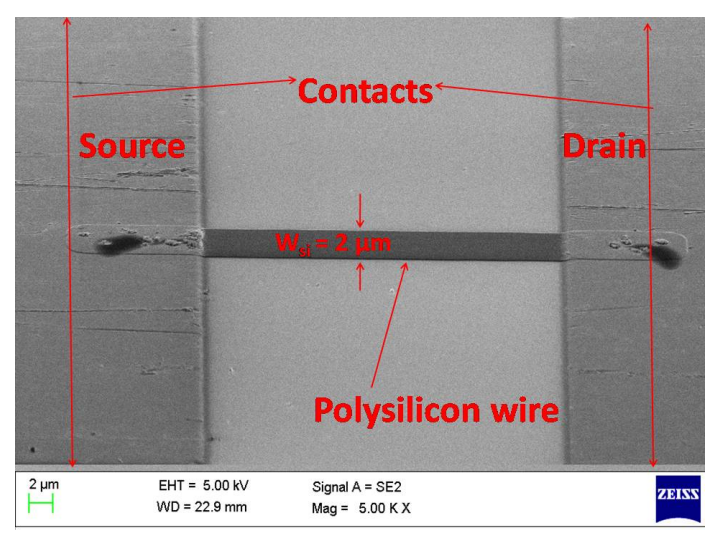

(b)

Figure 1: (a) Sheet resistance measurements through four-point-probe method. (b) SEM micrograph images of device fabricated.

$60 \mathrm{~nm}$ of $\mathrm{Si}_{3} N_{4}$ was deposited by LPCVD (diffusion bay) at $800{ }^{\circ} \mathrm{C} .50 \mathrm{~nm}$ of polycrystalline silicon was deposited through CVD. The samples were put to annealing chamber for dopant activation followed by boron doping in the diffusion bay. The wafers were subjected to the first optical lithography for patterning of the polySi wire. Polysilicon wires were obtained through dry etching (Oxford plasma lab RIE-100). The buried insulator layers between polysilicon wire and substrate were inserted for back-gating [17]. A very thin (5 $\mathrm{nm}$ ) layer of $\mathrm{Al}_{2} \mathrm{O}_{3}$ was deposited through the atomic layer deposition method. Second level Photolithography followed by wet etching (BOE) of sensing film was done to pattern the $\mathrm{Al}_{2} \mathrm{O}_{3}$ film aligned on polySi wire. For contact pads, $150 \mathrm{~nm}$ of $\mathrm{Cr} / \mathrm{Au}$ was deposited through sputtering. Further, the third level lithography was performed through lift-off to obtain the source and drain contact pads. The samples were later kept in the furnace at $350{ }^{\circ} \mathrm{C}$ for obtaining the better metal- polySi contact. The $150 \mathrm{~nm}$ of the passivation layer $\left(\mathrm{SiO}_{2}\right)$ was deposited and patterned through PECVD and lithography (followed by BOE), respectively, to protect the source and drain from the solution under test. For back-gating, $150 \mathrm{~nm} \mathrm{Cr} / \mathrm{Au}$ was deposited on the backside of the wafer. Furthermore, a thin $\mathrm{Al}_{2} \mathrm{O}_{3}$ film was deposited on the top-side of the wafer through atomic layer deposition to prevent any contact of the solution with contact pads, for long time measurements, and to increase the shelf life of the device. Further, an open window in the gate region was formed with resisn apoxy for the confinement of the test solution [18].

\subsection{Device Structural Characterization}

The Polysilicon and sensing oxide film $\left(\mathrm{Al}_{2} \mathrm{O}_{3}\right)$ thickness were measured through ellipsometry and found to be $52.9 \mathrm{~nm}$ and $4.95 \mathrm{~nm}$, respectively. The sheet resistance polysilicon film was done by the four-point probe method on three points of the wafer and found to be $121.67,120.46$, and $120.24 \Omega$ /square is shown in Fig. 1a, confirms the uniform doping of the device layer. The top-view scan electron image of the sensor fabricated through CMOS-compatible process for experimental analysis is shown in Fig. 1b, shows the min- 


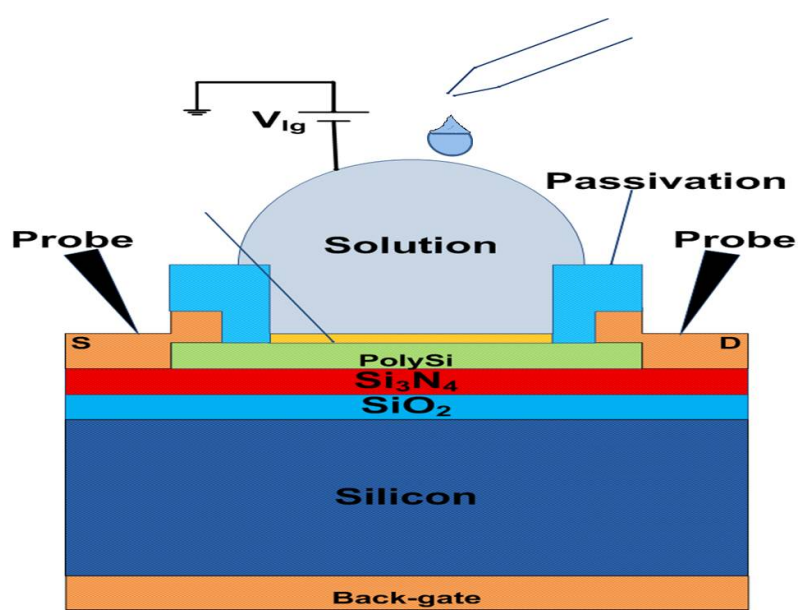

(a)

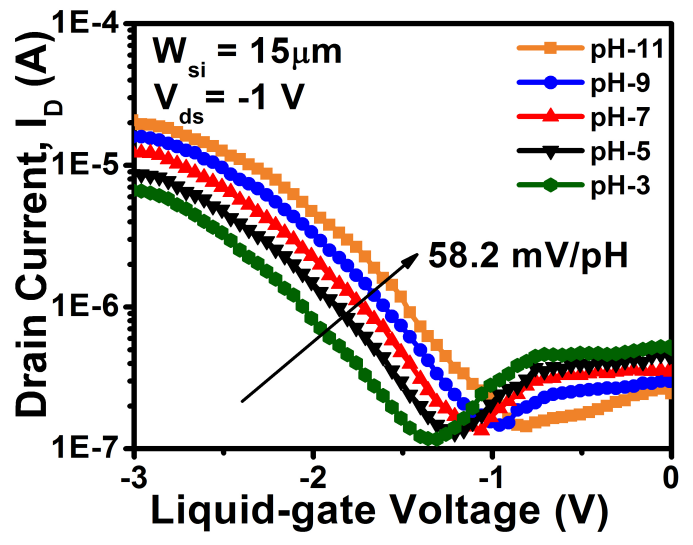

(b)

Figure 2: (a) Setup for characterization (b)Transfer characteristics for different pH of p-type JL-ISFET

imum channel width obtained was 2 micron. The fabricated wafer later kept in UV-O 3 ambiance for making the surface hydrophobic for the confinement of the test solution.

\subsection{Measurement setup of $\mathrm{pH}$ sensor}

The electrical measurements have been performed on the wafer level through a semiconductor parameter analyzer (Agilent B1500A). The micro-manipulators with needles were placed above sensing film over the channel region and a window was then etched to provide access to the contacts. The test $\mathrm{pH}$ solutions were drop-casted through a micro-pipette. The micro-pipette was attached with another manipulator's arm and placed over the sensing area. The micro-pipette was connected with the syringe through the silicone tubing. The syringe enables control over the flow of the test solution. All the measurements have been performed on device with channel width of $15 \mu \mathrm{m}$, as a maximum sensitivity (threshold voltage and drain current) on it have been reported in our previous work [16]. All the measurements have been performed in a dark environment to avoid the effect of light. The $\mathrm{pH}$ test solutions were prepared and calibrated through MK 6 digital $\mathrm{pH}$ meter.

\section{Results and discussion}

\subsection{Electrical characteristics of Junctionless ISFET}

To evaluate the sensing performance of p-channel JL-ISFET, the pH sensitivity measurement has been done through the shift in the threshold voltage of the device under different $\mathrm{pH}$ (3 to 11). The transfer characteristics have been obtained by sweeping the $V_{l g}$ from 0 to $-3 \mathrm{~V}$ and a low drain voltage $\left(V_{d s}\right)$ of $-1 \mathrm{~V}$ has been applied for the suppression of possible secondary effects [17]. It has been observed from Fig. $2 b$ that a decrease in hydrogen ion concentration at the interface results in a decrease in the threshold voltage (absolute value). Further, the JL-ISFET device exhibits a threshold voltage $\left(V_{t h}\right)$ sensitivity of $\sim 58.2$ $\mathrm{mV} / \mathrm{pH}$. It is very important to note that the drain current $\left(I_{D}\right)$ of ISFETs varies to some 
extent even in same pH solution. So in case of measurement of drain current $\left(I_{D}\right)$ shift only due to $\mathrm{pH}$ shift, it is very important to stabilize the device in the buffer solution before any measurement.

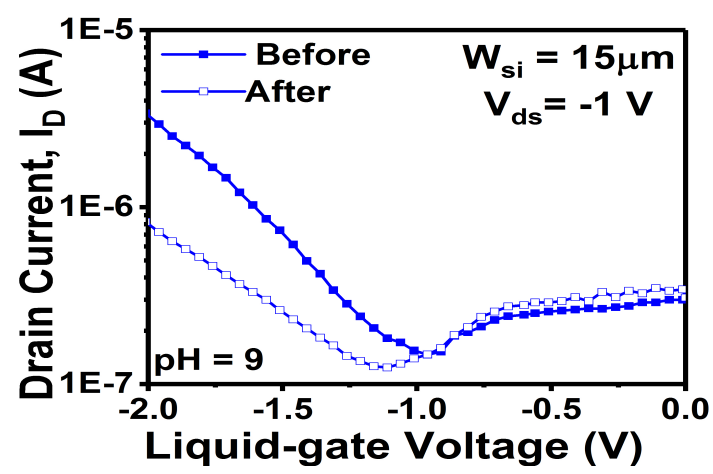

(a)

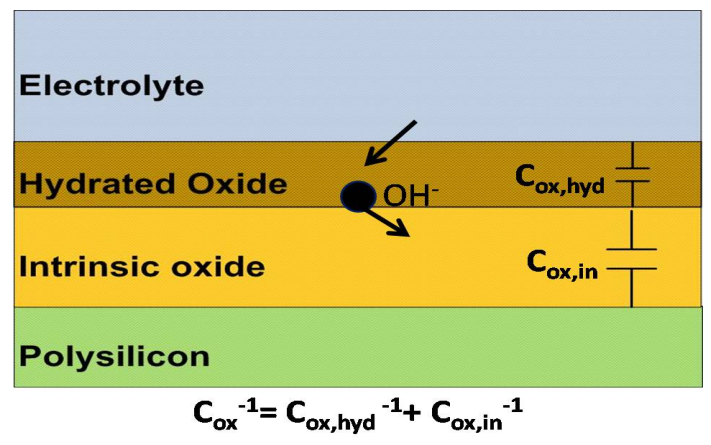

(b)

Figure 3: (a) Threshold voltage shift before and after transient measurements from the same device. (b) Surface reaction schematic and of composition of oxide capacitance.

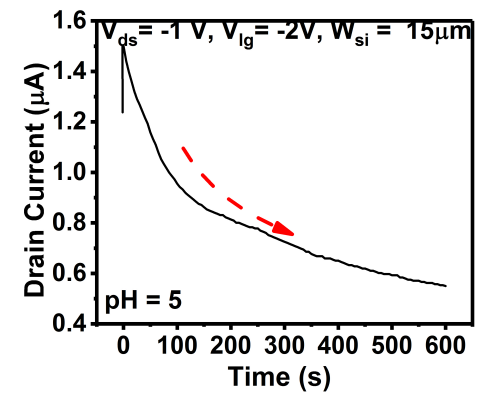

(a)

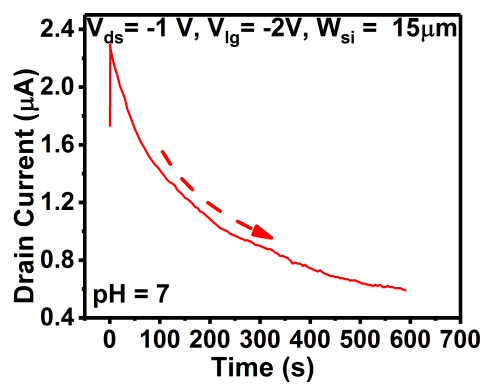

(b)

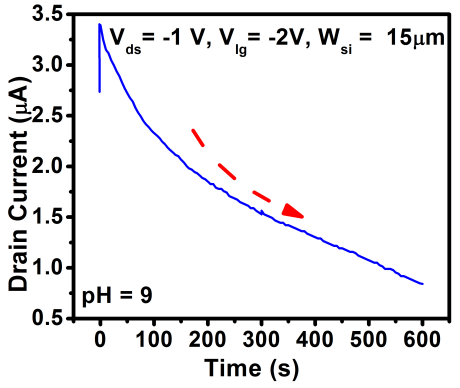

(c)

Figure 4: Transient characteristics of p-type JL-ISFET for (a) pH 5 (b) pH 7, and (c) pH 9.

\subsection{Drift analysis of junctionless ISFET}

To understand the drift phenomena in p-channel JL-ISFET, the transfer characteristics have been analyzed before and after the transient analysis under fix condition $\left(\mathrm{pH}=9, V_{l g}\right.$ $=-2 \mathrm{~V}$, and $V_{d s}=-1 \mathrm{~V}$ ) as shown in Fig. 3a. An evident drain current shift of $1.32 \mu \mathrm{A}$ $(\sim 63 \%)$ have been measured from the difference in the maximum drain current before and after transient analysis. The hydroxyl ions $\left(\mathrm{OH}^{-}\right)$contribute a major role and should be concerned for drain current drift in the p-channel JL-ISFET. However, the drift phenomena can not be described by the ionic diffusion because of very low mobility of hydroxyl ions through the sensing oxide film [19]. The drift in p-channel JL-ISFET can be explained through the modification (hydration) of sensing film surface due to its reaction with hydroxyl ion. 


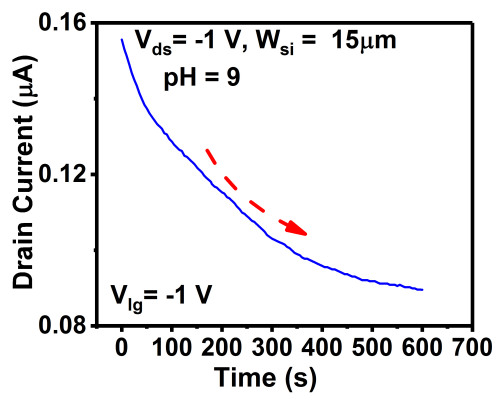

(a)

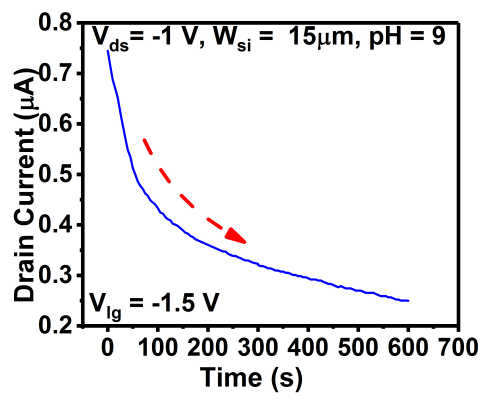

(b)

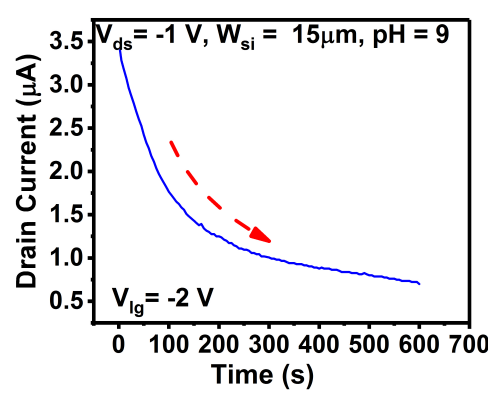

(c)

Figure 5: Transient characteristics of p-type JL-ISFET for (a) $V_{l g}=-1 \mathrm{~V}$ (b) $V_{l g}=-1.5 \mathrm{~V}$, and (c) $V_{l g}=$ $-2 \mathrm{~V}$.

It has also been explained in previous studies [20], [21] that the sensing film surface reaction with hydroxyl ions, results in its permanent dipole moment reduction that further reduces its capacitance $\left(C_{o x}\right)$. This effect can be represented as series combination of the capacitance of the oxide subjected to hydration $\left(C_{o x, h y d}\right)$ and the intrinsic (non-hydrated ) oxide capacitance $\left(C_{o x, \text { int }}\right)$, that can be seen in Fig. 3b.

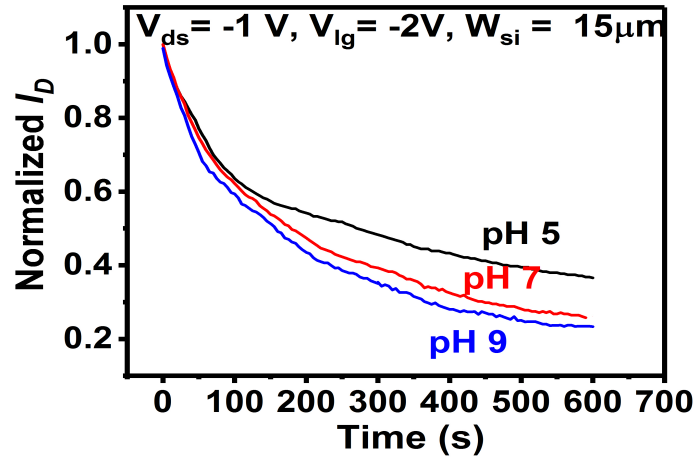

(a)

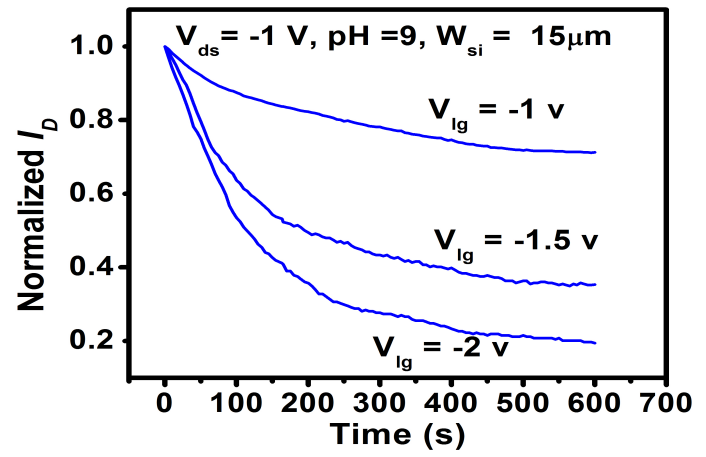

(b)

Figure 6: Transient characteristics of JL- ISFET for different (a) pH, and (b) Liquid-gate bias.

For further detailed investigation of the current drift phenomena in p-channel JL-ISFET, the transient drain current measurements have been performed for different $\mathrm{pH}$ and $V_{l g}$ as shown in Fig. 3 and Fig. 4 respectively. It has been observed from figures that under fixed bias conditions $\left(V_{l g}=-2, V_{d s}=-1 \mathrm{~V}\right.$, and $\left.\mathrm{pH}=5,7,9\right)$ and $\left(\mathrm{pH}=9, V_{d s}=-1 \mathrm{~V}\right.$, and $V_{l g}$ $=-2$ to $-1 \mathrm{~V})$, the drain current of the device decreases throughout the transient analysis as shown in Fig. 4a, 4b, 4c and Fig. 5a, 5b, 5c respectively. It has also been observed that there is an increase in the current drift with an increase in $\mathrm{pH}$ and supplied $V_{l g}$. Therefore, distinct devices with same dimensions have been used for characterization at each $\mathrm{pH}$ and $V_{l g}$ due to the continuous degradation of drain current. 
Further, for comparing the effect of $\mathrm{pH}$ and $V_{l g}$ on the current drift, the drain current has been normalized $\left(I_{D} / I_{D \text {,initial }}\right)$ during the measurement time as shown in Fig. 6a and 6b, respectively. A drain current drift of $75.2 \%, 73.8 \%$, and $63.8 \%$ has been measured for $\mathrm{pH} 9$, 7 , and 5 respectively, as shown in Fig. $7 \mathrm{~b}$. In addition, a current drift of $79.3 \%, 66.2 \%$, and $41.9 \%$ have been measured for $V_{l g}-2 \mathrm{~V},-1.5 \mathrm{~V}$, and $-1 \mathrm{~V}$, respectively, as shown in Fig. 7a. From the measured current drifts, it has been observed that the drift increases with $\mathrm{pH}$ as the hydroxyl ions increases towards the base-side $\mathrm{pH}$ cycle [22].

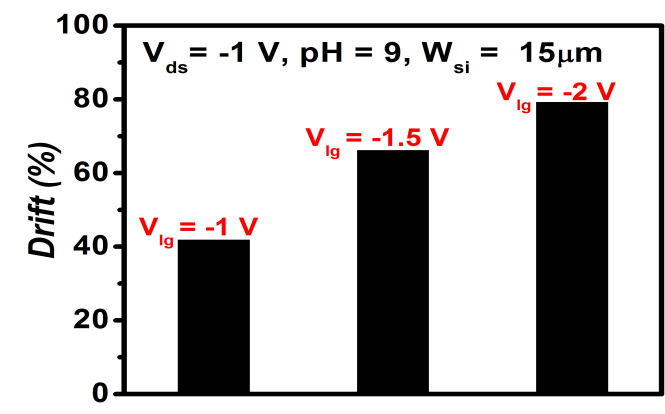

(a)

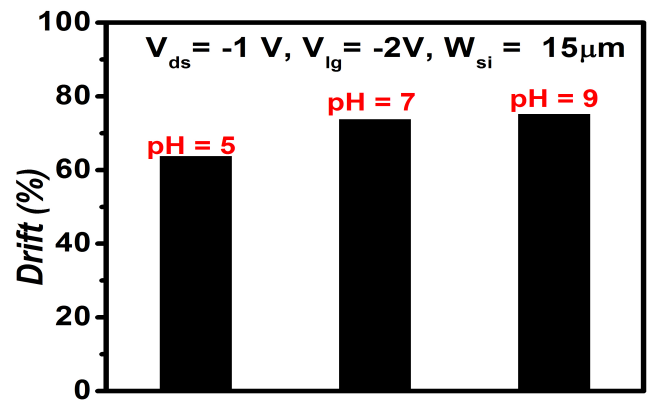

(b)

Figure 7: Percentage drift of junctionless ISFET for different (a) pH buffer, and (b) Liquid-gate voltages

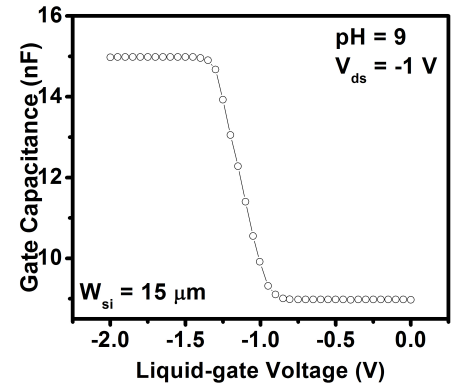

(a)

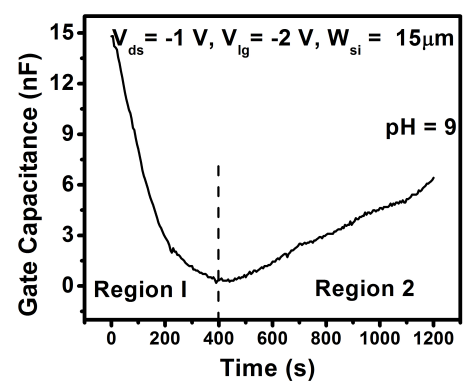

(b)

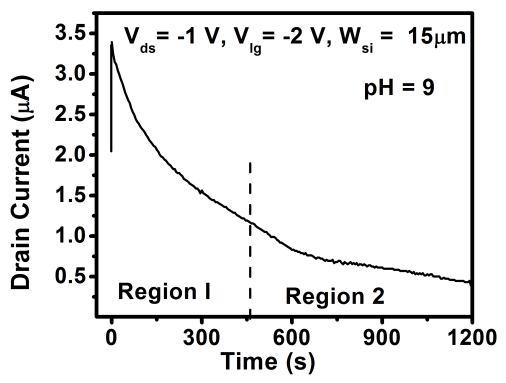

(c)

Figure 8: (a) $C_{G}-V_{l g}$ characteristics, (b) transient $C_{G}$ characteristics, and (c) the transient $I_{D}$ characteristics of p-channel JL-ISFET with identical dimensions and bias conditions.

The capacitive behavior of the p-channel JL-ISFET has also been analyzed for further analysis of current degradation. The gate capacitance $\left(C_{G}-V_{l g}, C_{G}-t\right)$ characteristics of the device have been demonstrated in Fig. 8a. and Fig. 8b, respectively. Fig. 8c shows the time-dependent drain current (with similar conditions as in Fig. 8b) for a longer evaluation period of 1200 seconds. It is observed from Fig. 8b that an increase in capacitance in region 2 , followed by a decrease in region 1 . The capacitance change in region 1 is related to the decrease in dielectric permittivity of the sensing film due to its chemical modification 


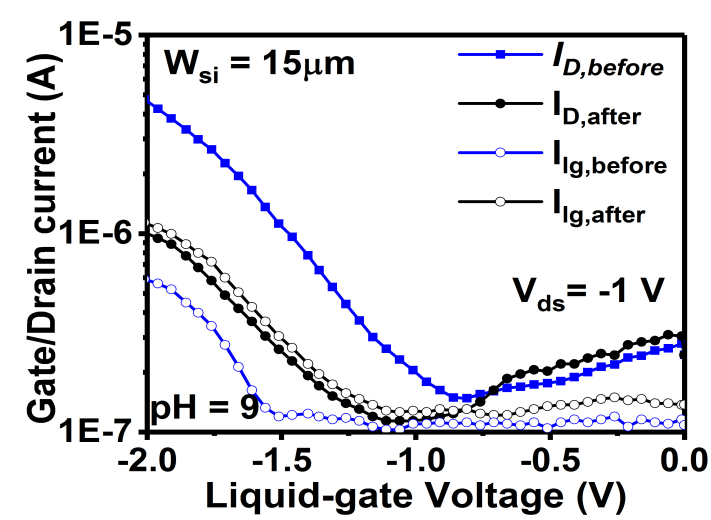

(a)

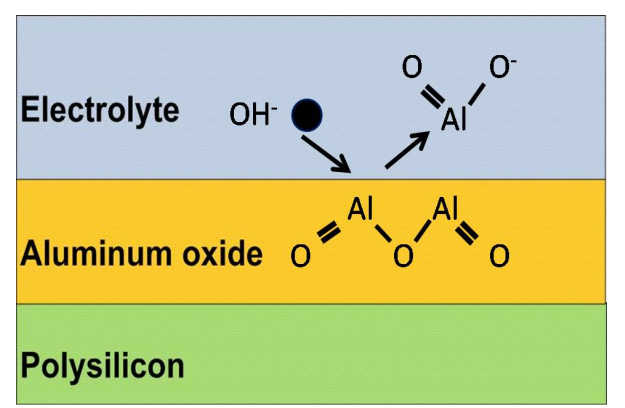

Dielectric etch : $-\mathrm{Al}_{2} \mathrm{O}_{3}+2 \mathrm{OH} \longrightarrow 2 \mathrm{AlO}_{2}+\mathrm{H}_{2} \mathrm{O}$

(b)

Figure 9: (a) $I_{D}$ and $I_{G^{-}} V_{l g}$ characteristics of the same device used to obtain the inset of Fig. 9 (b) at before and after the transient measurement (30 min), and (b) Schematic image of oxide etch induced by $O H^{-}$ion.

(hydration), and continuous capacitance increase in region 2 may be due to etching of the sensing film by hydroxyl ions, which results in a continuous decay in the drain current.

The drain current decay phenomena has been further analyzed by measuring the gate leakage current $\left(I_{G}\right)$ with respect to the $V_{l g}$ before and after the transient measurement. A noticeable increase of in gate leakage fter the transient analysis can be seen from Fig. 9a. The decrease of drain current $\left(I_{D}\right)$ despite an increase in gate capacitance (Fig. 8b and Fig. 8c) may be due to an increase in gate leakage current. A decrease in oxide film thickness $\left(t_{o x}\right)$ due to its etching by exposing it with hydroxyl ions for long time, may also be the cause of an increase in gate leakage current. The etching phenomena is similar to $\left(\mathrm{NH}_{4} \mathrm{OH} / \mathrm{H}_{2} \mathrm{O}_{2} / \mathrm{H}_{2} \mathrm{O}\right)$ mixture (SC 1) cleaning method (Fig. 9b). It is also worth mentioning that, the drain current decay is indefinite for p-channel JL-ISFET.

\subsection{Hysteresis effect Analysis}

The hysteresis width of the p-channel JL-ISFET are measured between the first output voltage and final output voltage for same $\mathrm{pH}$ value. The hysteresis behavior was observed for different $\mathrm{pH}$ buffer solutions with the $\mathrm{pH}$ loop cycle of $7 \rightarrow 3 \rightarrow 7 \rightarrow 11 \rightarrow 7$ (acid-side) and $7 \rightarrow 11 \rightarrow 7 \rightarrow 3 \rightarrow 7$ (base-side) for 960 seconds. The measured hysteresis widths were 1.3 , and $5.7 \mathrm{mV}$ for acid-side and base-side, respectively. The difference in hysteresis width with $\mathrm{pH}$ loop cycles is due to the different size of $\mathrm{H}^{+}$and $\mathrm{OH}^{-}$ions, the diffusion of $\mathrm{H}^{+}$ ions to the buried site is faster than the diffusion of $\mathrm{OH}^{-}$ions [23].

Further, the hysteresis width for different loop time (960 s, $1500 \mathrm{~s}$, and $1920 \mathrm{~s})$ has also been investigated. The hysteresis width for the $\mathrm{pH}$ loop path of $7 \rightarrow 3 \rightarrow 7 \rightarrow 11$ $\rightarrow 7$ were measured and found to be $1.3,3.4$, and $6.7 \mathrm{mV}$ for 960,1500 , and 1920 second, respectively as shown in Fig. 10b. It can be inferred from the hysteresis results that the hysteresis width increases with loop time as more hydration and etching of the sensing film occurs for more loop time. Table 2 represents the hysteresis widths of p-channel JL-ISFET comparison with the state of art devices with different sensing films. The data from the 


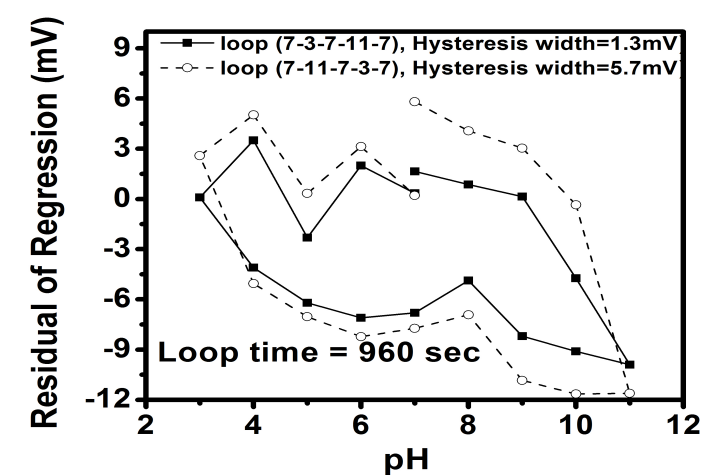

(a)

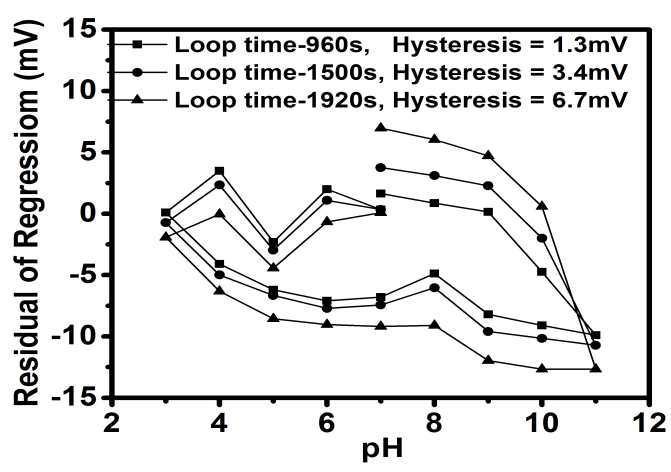

(b)

Figure 10: Residual plot of hysteresis for different (a) pH loop path, and (b) Loop time

table demonstrates that the p-channel JL-ISFET shows improved hysteresis behavior than previously reported devices. Furthermore, the p-channel JL-ISFET has lower hysteresis width than the conventional $\mathrm{Al}_{2} \mathrm{O}_{3}$ gate ISFET [23], which may be due to the better quality (less porosity) of the ALD deposited $\mathrm{Al}_{2} \mathrm{O}_{3}$ in p-channel JL-ISFET.

Table 2: Comparison of the hysteresis of the proposed device and state-of-the-art devices.

\begin{tabular}{lll}
\hline Sensing Film & Hysteresis $(\mathbf{m V})$ & Loop Time (s) \\
\hline \hline $\mathrm{WO}_{3}[18]$ & 15.7 & 1500 \\
\hline $\mathrm{Ai}_{2} \mathrm{O}_{3}[23]$ & 4 & 960 \\
\hline $\mathrm{Si}_{3} \mathrm{~N}_{4}[24]$ & 2 & 960 \\
\hline $\mathrm{Tm}_{2} \mathrm{Ti}_{2} \mathrm{O}_{7}[25]$ & 4.3 & 1500 \\
\hline $\mathrm{Al}_{2} \mathrm{O}_{3}$ (This device) & $1.3,3.4$ & 960,1500 \\
\hline
\end{tabular}

\section{Conclusion}

The drain current drift phenomena and hysteresis mechanism of p-channel JL-ISFET have been experimentally investigated for the first time. It has been observed that sensing film hydration and thickness reduction through hydroxyl ions, are the dominating factors for drift and hysteresis. Further, it has also been observed that the current drift increases with $\mathrm{pH}$ and liquid-gate voltage $\left(V_{l g}\right)$ as the hydroxyl ions activity increases towards the base-side. A maximum drain current drift of $75.2 \%$ and $79.3 \%$ have been measured for $\mathrm{pH}$ $=9$ and $V_{l g}=-2 \mathrm{~V}$, respectively. Furthermore, the hysteresis behavior analysis of p-channel JL-ISFET has also been performed for different $\mathrm{pH}$ loop paths and loop times. It has also been observed from the hysteresis characteristics that hysteresis width increases with the $\mathrm{pH}$ loop-time and pH loop-cycle towards the base-side. 


\section{Declaration}

\subsection{Ethics Approval and consent to participate}

Not Applicable.

\subsection{Consent for publication}

Not Applicable.

\subsection{Availability of data and Materials}

The datasets generated during and/or analysed during the current study are available from the corresponding author on reasonable request.

\subsection{Competing Interests}

The authors have no relevant financial or non-financial interests to disclose.

\subsection{Funding}

Authors would like to thank CENSE, Indian Institute of Science, Bengaluru for providing the fabrication and characterization facility under the Indian nanoelectronic user's program (INUP).

\subsection{Author's Contribution}

Jaydeep SIngh parmar, Nawaz Shafi, and Chitrakant Sahu conceived this research and designed experiments, jaydeep singh parmar wrote the paper and participated in the design and interpretation of the data, Jaydeep SIngh parmar, Nawaz Shafi, and Chitrakant Sahu participated in the revisions of it.

\subsection{Acknowledgments}

Authors acknowledge CENSE, Indian Institute of Science, Bengaluru for providing the fabrication and characterization facility under the Indian nanoelectronic user's program (INUP), and department of Electronics and Communication Engineering, MNIT Jaipur to use their resources and support.

\subsection{Author's Information}

The name(s) of the author(s) - Jaydeep SIngh parmar, Nawaz Shafi, and Chitrakant Sahu. The affiliation(s) of the author(s)- Dept. of Elec. \& Commn., Malaviya National Institue of Technology, Jaipur, , (Rajasthan), India. Active e-mail address of the corresponding author-2016rec9510@mnit.ac.in. ORCID ID of the author- 0000-0002-0424-8703.

\subsection{Disclosure of potential Conflicts of Intrest}

The authors declare that they have no conflict of interest.

\subsection{Research Involving Human Participants and/or Animals}

Not Applicable. 


\subsection{Informed Consent Not Applicable. ,}

[1] V. Mani, B. Devadas, S.-M. Chen, Direct electrochemistry of glucose oxidase at electrochemically reduced graphene oxide-multiwalled carbon nanotubes hybrid material modified electrode for glucose biosensor, Biosensors and Bioelectronics 41 (2013) 309-315.

[2] A. T. Sage, J. D. Besant, B. Lam, E. H. Sargent, S. O. Kelley, Ultrasensitive electrochemical biomolecular detection using nanostructured microelectrodes, Accounts of chemical research 47 (8) (2014) 24172425 .

[3] C. Zhu, Z. Zeng, H. Li, F. Li, C. Fan, H. Zhang, Single-layer mos2-based nanoprobes for homogeneous detection of biomolecules, Journal of the American Chemical Society 135 (16) (2013) 5998-6001.

[4] N. Chartuprayoon, M. Zhang, W. Bosze, Y.-H. Choa, N. V. Myung, One-dimensional nanostructures based bio-detection, Biosensors and Bioelectronics 63 (2015) 432-443.

[5] W.-Y. Lee, S.-R. Kim, T.-H. Kim, K. S. Lee, M.-C. Shin, J.-K. Park, Sol-gel-derived thick-film conductometric biosensor for urea determination in serum, Analytica Chimica Acta 404 (2) (2000) 195-203.

[6] J.-H. Ahn, J.-Y. Kim, M.-L. Seol, D. J. Baek, Z. Guo, C.-H. Kim, S.-J. Choi, Y.-K. Choi, A ph sensor with a double-gate silicon nanowire field-effect transistor, Applied Physics Letters 102 (8) (2013) 083701.

[7] A. Gao, N. Lu, P. Dai, T. Li, H. Pei, X. Gao, Y. Gong, Y. Wang, C. Fan, Silicon-nanowire-based cmoscompatible field-effect transistor nanosensors for ultrasensitive electrical detection of nucleic acids, Nano letters 11 (9) (2011) 3974-3978.

[8] F. Patolsky, G. Zheng, C. M. Lieber, Fabrication of silicon nanowire devices for ultrasensitive, label-free, real-time detection of biological and chemical species, Nature protocols 1 (4) (2006) 1711.

[9] S. J. Kim, J. Jung, K. W. Lee, D. H. Yoon, T. S. Jung, S. R. Dugasani, S. H. Park, H. J. Kim, Low-cost label-free electrical detection of artificial dna nanostructures using solution-processed oxide thin-film transistors, ACS applied materials \& interfaces 5 (21) (2013) 10715-10720.

[10] Z. J. Huang, Real-time profitability of published anomalies: An out-of-sample test, Available at SSRN 1364813.

[11] J.-P. Colinge, C.-W. Lee, A. Afzalian, N. D. Akhavan, R. Yan, I. Ferain, P. Razavi, B. O'neill, A. Blake, M. White, et al., Nanowire transistors without junctions, Nature nanotechnology 5 (3) (2010) 225-229.

[12] C. Sahu, J. Singh, Charge-plasma based process variation immune junctionless transistor, IEEE Electron Device Letters 35 (3) (2014) 411-413.

[13] R. Narang, M. Saxena, M. Gupta, Analytical model of ph sensing characteristics of junctionless silicon on insulator isfet, IEEE Transactions on Electron Devices 64 (4) (2017) 1742-1750.

[14] N. Shafi, C. Sahu, C. Periasamy, Fabrication and ph sensitivity analysis of in-situ doped polycrystalline silicon thin-film junctionless biofet, IEEE Electron Device Letters 40 (6) (2019) 997-1000.

[15] E. Buitrago, G. Fagas, M. F.-B. Badia, Y. M. Georgiev, M. Berthomé, A. M. Ionescu, Junctionless silicon nanowire transistors for the tunable operation of a highly sensitive, low power sensor, Sensors and Actuators B: Chemical 183 (2013) 1-10.

[16] J. S. Parmar, C. Sahu, Cmos-compatible ex-situ incorporated junctionless enhancement-mode thin polysilicon film fet ph sensor, IEEE Transactions on Device and Materials Reliability.

[17] H. Jang, J. Lee, J. Han Lee, S. Seo, B.-G. Park, D. Myong Kim, D. Hwan Kim, I.-Y. Chung, Analysis of hysteresis characteristics of silicon nanowire biosensors in aqueous environment, Applied Physics Letters 99 (25) (2011) 252103.

[18] J.-L. Chiang, S.-S. Jan, J.-C. Chou, Y.-C. Chen, Study on the temperature effect, hysteresis and drift of ph-isfet devices based on amorphous tungsten oxide, Sensors and Actuators B: Chemical 76 (1-3) (2001) 624-628.

[19] S. Jamasb, S. D. Collins, R. L. Smith, A physically-based model for drift in al/sub 2/o/sub 3/-gate ph isfet's, in: Proceedings of International Solid State Sensors and Actuators Conference (Transducers' 97), Vol. 2, IEEE, 1997, pp. 1379-1382.

[20] S. Jamasb, S. D. Collins, R. L. Smith, A physical model for threshold voltage instability in si/sub 3/n/sub 4/-gate h/sup+/-sensitive fet's (ph isfet's), IEEE Transactions on Electron Devices 45 (6) (1998) 1239-1245. 
[21] S. Jamasb, S. Collins, R. L. Smith, A physical model for drift in ph isfets, Sensors and Actuators B: Chemical 49 (1-2) (1998) 146-155.

[22] J.-L. Chiang, J.-C. Chou, Y.-C. Chen, G. S. Liau, C.-C. Cheng, Drift and hysteresis effects on aln/sio2 gate ph ion-sensitive field-effect transistor, Japanese journal of applied physics 42 (8R) (2003) 4973.

[23] J.-C. Chou, C.-Y. Weng, Sensitivity and hysteresis effect in al2o3 gate ph-isfet, Materials chemistry and physics 71 (2) (2001) 120-124.

[24] L. Bousse, S. Mostarshed, B. van der Schoot, N. De Rooij, Comparison of the hysteresis of ta2o5 and si3n4 ph-sensing insulators, Sensors and Actuators B: Chemical 17 (2) (1994) 157-164.

[25] T. Pan, C. Lin, W. Lin, M. Wu, High- $\kappa \mathrm{tm}_{2} \mathrm{ti}_{2} \mathrm{O}_{7}$ electrolyte-insulator-semiconductor creatinine biosensor, IEEE Sensors Journal 11 (10) (2011) 2388-2394. doi:10.1109/JSEN.2011.2122255. 